\title{
An Inverse Spectral Problem for the Matrix Sturm-Liouville Operator with a Bessel-Type Singularity
}

\author{
Natalia Bondarenko \\ Department of Mechanics and Mathematics, Saratov State University, Astrakhanskaya 83, Saratov 410012, Russia \\ Correspondence should be addressed to Natalia Bondarenko; bondarenkonp@info.sgu.ru
}

Received 21 September 2014; Accepted 10 May 2015

Academic Editor: Salim Messaoudi

Copyright @ 2015 Natalia Bondarenko. This is an open access article distributed under the Creative Commons Attribution License, which permits unrestricted use, distribution, and reproduction in any medium, provided the original work is properly cited.

\begin{abstract}
The inverse problem by the Weyl matrix is studied for the matrix Sturm-Liouville equation on a finite interval with a Besseltype singularity in the end of the interval. We construct special fundamental systems of solutions for this equation and prove the uniqueness theorem of the inverse problem.
\end{abstract}

\section{Introduction}

Inverse problems of the spectral analysis for systems of differential equations with Bessel-type singularities arise in quantum mechanics (e.g., in the theory of the deuteron [1]). Although inverse problems for scalar operators with singularities have been studied for a long time [2-4], there are no general results for matrix operators. In the monograph [1], the inverse scattering problem is studied for some particular cases of matrix Sturm-Liouville equations with singularities. In this paper, we start a research on inverse spectral problems for systems with Bessel-type singularities. We suggest an approach, based on the method of spectral mappings [5]. One of the main ingredients of our method is special fundamental systems of solutions (FSS): Besseltype solutions with the prescribed asymptotic behavior at the singular point and Birkhoff-type solutions with the known asymptotic representations, as the spectral parameter tends to infinity. The connection between these two FSS plays the most important role in the investigation. In this paper, we formulate an inverse problem by the Weyl matrix for the matrix Sturm-Liouville operator with a singularity and prove the uniqueness theorem.

Consider the matrix Sturm-Liouville equation on a finite interval with a Bessel-type singularity in the end of the interval

$$
\ell(Y):=-Y^{\prime \prime}+\left(\frac{\omega}{x^{2}}+Q(x)\right) Y=\lambda Y, \quad x \in(0, T) .
$$

Here $Y=\left[y_{k}(x)\right]_{k=1}^{m}$ is a vector function, $\lambda$ is the spectral parameter, and $Q(x)$ and $\omega$ are $m \times m$ matrices.

We assume that the matrix $\omega$ is diagonal; that is, $\omega=$ $\operatorname{diag}\left\{\omega_{1}, \omega_{2}, \ldots, \omega_{m}\right\}, \omega_{q} \in \mathbb{R}, q=\overline{1, m}$. If $\omega$ is an arbitrary Hermitian matrix, one can apply the standard unitary transform, in order to fulfill this condition. For definiteness, let $\omega_{q}=v_{q}^{2}-1 / 4, v_{1} \geq v_{2} \geq \cdots \geq v_{m}>0, v_{q} \notin \mathbb{N}, q=\overline{1, m}$. Let the matrix function $x^{1-2 v_{1}} Q(x)$ be integrable on $(0, T)$.

\section{Bessel-Type Solutions}

Let $\rho=\sqrt{\lambda}, \arg \rho \in[0, \pi)$. If we put $Q(x) \equiv 0,(1)$ splits into $m$ scalar equations, which have Bessel solutions [6]:

$$
c_{j q}(x, \lambda)=x^{\mu_{j q}} \sum_{k=0}^{\infty} c_{j k q}(\rho x)^{2 k}, \quad j=1,2, q=\overline{1, m},
$$

where

$$
\begin{aligned}
& \mu_{1 q}=\frac{1}{2}-v_{q}, \\
& \mu_{2 q}=\frac{1}{2}+v_{q}, \\
& c_{10 q} c_{20 q}=\frac{1}{2 v_{q}},
\end{aligned}
$$




$$
\begin{aligned}
& c_{j k q}=(-1)^{k} \\
& \cdot c_{j 0 q}\left(\prod_{s=1}^{k}\left(\left(2 s+\mu_{j q}\right)\left(2 s+\mu_{j q}-1\right)-\omega_{q}\right)\right)^{-1} .
\end{aligned}
$$

Clearly, the matrix functions $C_{j}(x, \lambda)=\operatorname{diag}\left\{c_{j q}(x, \lambda)\right\}_{q=1}^{m}$ satisfy (1).

Let $S_{j}(x, \lambda)$ and $S_{j}^{*}(x, \lambda), j=1,2$, be the matrix solutions of the following integral equations:

$$
\begin{aligned}
& S_{j}(x, \lambda)=C_{j}(x, \lambda)-\int_{0}^{x} G(x, t, \lambda) Q(t) S_{j}(t, \lambda) d t, \\
& S_{j}^{*}(x, \lambda)=C_{j}(x, \lambda)-\int_{0}^{x} S_{j}^{*}(t, \lambda) Q(t) G(x, t, \lambda) d t,
\end{aligned}
$$

where $G(x, t, \lambda)=C_{2}(x, \lambda) C_{1}(t, \lambda)-C_{1}(x, \lambda) C_{2}(t, \lambda)$.

Along with (1), consider the following equation:

$$
-Z^{\prime \prime}+Z\left(\frac{\omega}{x^{2}}+Q(x)\right)=\lambda Z, \quad x \in(0, T),
$$

where $Z=Z(x)$ is a row vector.

Denote the unit $m \times m$ matrix by $I$, its $q$ th column by $e_{q}$, and its $q$ th row by $e_{q}^{*}$. The column vectors $S_{j}(x, \lambda) e_{q}, j=1,2$, $q=\overline{1, m}$, form a fundamental system of solutions (FSS) of (1). Similarly the row vectors $e_{q}^{*} S_{j}^{*}(x, \lambda)$ form an FSS of (5). Clearly, for each fixed $x \in(0, T)$, the matrix functions $S_{j}^{(\nu)}(x, \lambda), S_{j}^{*(\nu)}(x, \lambda)$ are entire in the $\lambda$-plane. Using integral equations (4) and the integrability of the matrix function $x^{1-2 v_{1}} Q(x)$, one obtains the following asymptotic formulas as $x \rightarrow 0, j=1,2$, and $q=\overline{1, m}$ :

$$
\begin{aligned}
& S_{j}(x, \lambda) e_{q}=O\left(x^{\mu_{j q}}\right) \text {, } \\
& e_{q}^{*} S_{j}^{*}(x, \lambda)=O\left(x^{\mu_{j q}}\right), \\
& x^{-\mu_{j q}}\left(S_{j}(x, \lambda)-C_{j}(x, \lambda)\right) e_{q}=o\left(x^{2 v_{1}}\right) \text {, } \\
& x^{-\mu_{j q}} e_{q}^{*}\left(S_{j}^{*}(x, \lambda)-C_{j}(x, \lambda)\right)=o\left(x^{2 v_{1}}\right) .
\end{aligned}
$$

Denote $\langle Z, Y\rangle:=Z^{\prime} Y-Y Z^{\prime}$. It is easy to show that if the vector functions $Y(x)$ and $Z(x)$ satisfy (1) and (5), respectively, then $\langle Z(x), Y(x)\rangle$ does not depend on $x$. Note that $C_{j}(x, \lambda), j=1,2$, are the matrix solutions of (5) for $Q(x) \equiv 0$. Moreover, $\left\langle C_{j}(x, \lambda), C_{k}(x, \lambda)\right\rangle=(-1)^{j} \delta_{j k} I, j, k=$ 1,2 , where $\delta_{j k}$ is the Kronecker delta. Using this fact together with asymptotic formulas (6), we obtain the relation

$$
\left\langle S_{j}^{*}(x, \lambda), S_{k}(x, \lambda)\right\rangle=(-1)^{j} \delta_{j k} I, \quad j, k=1,2 .
$$

\section{Birkhoff-Type Solutions}

Following ideas of [3], we construct the solutions $Y_{k}(x, \rho)$, $k=1,2$, whose columns form an FSS of (1) and have the following properties. $\left(i_{1}\right)$ For each fixed $x \in(0, T)$, the matrix functions $Y_{k}^{(v)}(x, \rho), k=1,2, \nu=0,1$, are analytic in the set $\Omega:=\{\rho$ : $\left.\operatorname{Im} \rho>0,|\rho|>\rho^{*}\right\}$ and continuous in $\bar{\Omega}$.

$\left(i_{2}\right)$ For $x \in(0, T), \rho \in \bar{\Omega},|\rho x| \geq 1$, and $\nu=0,1$, the following asymptotic formulas are valid:

$$
\begin{aligned}
& Y_{1}^{(v)}(x, \rho)=(i \rho)^{\nu} \exp (i \rho x)[I]_{\beta}, \\
& Y_{2}^{(v)}(x, \rho)=(-i \rho)^{v} \exp (-i \rho x)[I]_{\beta},
\end{aligned}
$$

where $[I]_{\beta}=\left(I+O\left(\rho^{-\beta}\right)\right), \beta=\min \left\{1,2 \nu_{1}\right\}$.

$\left(i_{3}\right)$ The solutions $S_{j}(x, \lambda)$ and $Y_{k}(x, \lambda)$ are connected by the relations

$$
Y_{k}(x, \lambda)=\sum_{j=1}^{2} S_{j}(x, \lambda) B_{k j}(\rho),
$$

and the Stokes multipliers $B_{k j}(\rho)$ have the following asymptotic behavior:

$$
B_{k j}(\rho)=\operatorname{diag}\left\{\rho^{\mu_{j q}}\right\} B_{k j}^{0}[I]_{\beta}, \quad k, j=1,2, q=\overline{1, m},
$$

where $B_{k j}^{0}=\operatorname{diag}\left\{\beta_{k j q}\right\}$ and $\beta_{k j q}$ are constant Stokes multipliers for solutions of the scalar equations $-y^{\prime \prime}+\left(\omega_{q} / x^{2}\right) y=y$ (see [3]).

Expansion (9) and asymptotic formula (10) play a crucial role in the study of the inverse problem. Using (9) and (10), we derive asymptotic formulas for $S_{j}(x, \lambda)$ :

$$
\begin{aligned}
S_{j}^{(\nu)} & (x, \lambda) \\
& =\frac{i(-1)^{j}}{2}(-i \rho)^{\nu} \exp (-i \rho x)[I]_{\beta} \operatorname{diag}\left\{\rho^{-\mu_{j q}}\right\} B_{1,3-j}^{0}
\end{aligned}
$$

for $\rho \in \Omega_{\delta}:=\{\rho \in \Omega: \delta<\arg \rho<\pi-\delta\}, \delta>0$, $\nu=0,1$, and $x \in(0, T)$. Similarly, Birkhoff-type solutions can be constructed for (5), and the asymptotic formulas for the solutions $S_{j}^{*}(x, \lambda)$ can be obtained.

\section{Inverse Problem}

Introduce the linear forms $\sigma_{1}(Y):=\left\langle S_{2}^{*}(x, \lambda), Y\right\rangle, \sigma_{2}(Y):=$ $-\left\langle S_{1}^{*}(x, \lambda), Y\right\rangle$. In view of (7), we have $\sigma_{j}\left(S_{k}\right)=\delta_{j k} I$. Note that for the classical matrix Sturm-Liouville equation we have $\sigma_{1}(Y)=Y(0), \sigma_{2}(Y)=Y^{\prime}(0)$.

Consider the boundary value problem $L=L(Q, h, H)$ for (1) with the boundary conditions

$$
\begin{aligned}
& U(Y):=\sigma_{2}(Y)-h \sigma_{1}(Y)=0, \\
& V(Y):=Y^{\prime}(T)+H Y(T)=0,
\end{aligned}
$$

where $h$ and $H$ are $m \times m$ matrices. One can also take the Dirichlet-type boundary condition $\sigma_{1}(Y)=0$ at $x=0$. If $v_{m} \geq 1 / 2$, it is equivalent to the standard Dirichlet boundary condition $Y(0)=0$. Similarly, one can investigate the matrix Sturm-Liouville equation with Bessel-type singularities at both ends of the interval. Then both boundary conditions take the form similar to (12). 
Let $\Phi(x, \lambda)$ be the matrix solution of (1), satisfying the conditions $U(\Phi)=I, V(\Phi)=0$. The matrix function $M(\lambda):=$ $\Phi(0, \lambda)$ is called the Weyl matrix of the problem $L$. The Weyl matrix generalizes the notion of the Weyl function for the scalar case $m=1$ (see $[5,7])$.

Let $\varphi(x, \lambda)$ be the matrix solution of (1) under initial conditions $\sigma_{1}(\varphi)=I, \sigma_{2}(\varphi)=h$. Obviously,

$$
\begin{aligned}
& \varphi(x, \lambda)=S_{1}(x, \lambda)+S_{2}(x, \lambda) h, \\
& \Phi(x, \lambda)=S_{2}(x, \lambda)+\varphi(x, \lambda) M(\lambda) .
\end{aligned}
$$

Introduce the linear forms $\sigma_{1}^{*}(Z):=-\left\langle Z, S_{2}(x, \lambda)\right\rangle$, $\sigma_{2}^{*}(Z):=\left\langle Z, S_{1}(x, \lambda)\right\rangle$ and consider the boundary value problem $L^{*}=L^{*}(Q, h, H)$ for (5) with the boundary conditions

$$
\begin{aligned}
& U^{*}(Z):=\sigma_{2}^{*}(Z)-\sigma_{1}^{*}(Z) h=0, \\
& V^{*}(Z):=Z^{\prime}(T)+Z(T) H=0 .
\end{aligned}
$$

Similarly one can define the problem $\widetilde{L}^{*}=L^{*}(\widetilde{Q}, \widetilde{h}, \widetilde{H})$. Let $\varphi^{*}(x, \lambda)$ and $\Phi^{*}(x, \lambda)$ be matrix solutions of $(5)$, satisfying the conditions $\varphi^{*}(0, \lambda)=I, \varphi^{* \prime}(0, \lambda)=h, U^{*}\left(\Phi^{*}\right)=I$, and $V^{*}\left(\Phi^{*}\right)=0$. Put $M^{*}(\lambda):=\Phi^{*}(0, \lambda)$. Similarly to (14), one can easily derive

$$
\begin{aligned}
& \varphi^{*}(x, \lambda)=S_{1}^{*}(x, \lambda)+h S_{2}^{*}(x, \lambda), \\
& \Phi^{*}(x, \lambda)=S_{2}^{*}(x, \lambda)+M^{*}(\lambda) \varphi^{*}(x, \lambda) .
\end{aligned}
$$

Note that the expressions $\left\langle\varphi^{*}(x, \lambda), \varphi(x, \lambda)\right\rangle,\left\langle\Phi^{*}(x, \lambda), \Phi(x\right.$, $\lambda)\rangle$ do not depend on $x$. Using relations (14), (16), and (7), we obtain

$$
\begin{aligned}
\left\langle\varphi^{*}(x, \lambda), \varphi(x, \lambda)\right\rangle & =0, \\
\left\langle\Phi^{*}(x, \lambda), \Phi(x, \lambda)\right\rangle & =M(\lambda)-M^{*}(\lambda) .
\end{aligned}
$$

In addition,

$$
\begin{aligned}
\left\langle\Phi^{*}(x, \lambda), \Phi(x, \lambda)\right\rangle= & \left\langle\Phi^{*}(x, \lambda), \Phi(x, \lambda)\right\rangle_{x=T} \\
= & V^{*}\left(\Phi^{*}\right) \Phi(T, \lambda) \\
& -\Phi^{*}(T, \lambda) V(\Phi)=0 .
\end{aligned}
$$

Hence $M(\lambda) \equiv M^{*}(\lambda)$.

We study the following inverse problem: given the Weyl matrix $M(\lambda)$, find $Q(x), h$, and $H$.

Consider the boundary value problem $\widetilde{L}=L(\widetilde{Q}, \widetilde{h}, \widetilde{H})$ in the same form as $L$, but with other coefficients. We agree that if a certain symbol $\gamma$ denotes an object related to $L$, then the corresponding symbol $\tilde{\gamma}$ with tilde denotes the analogous object related to $\widetilde{L}$.

Theorem 1. If $M(\lambda)=\widetilde{M}(\lambda)$, then $Q(x)=\widetilde{Q}(x)$ a.e. on $(0, T)$, $h=\widetilde{h}$, and $H=\widetilde{H}$. Thus, the boundary value problem $L$ can be determined by its Weyl matrix uniquely.
Proof. Define the block matrix $P(x, \lambda)=\left[P_{j k}(x, \lambda)\right]_{j, k=1,2}$ by the formula

$$
\begin{gathered}
P(x, \lambda)\left[\begin{array}{cc}
\widetilde{\varphi}(x, \lambda) & \widetilde{\Phi}(x, \lambda) \\
\widetilde{\varphi}^{\prime}(x, \lambda) & \widetilde{\Phi}^{\prime}(x, \lambda)
\end{array}\right] \\
=\left[\begin{array}{cc}
\varphi(x, \lambda) & \Phi(x, \lambda) \\
\varphi^{\prime}(x, \lambda) & \Phi^{\prime}(x, \lambda)
\end{array}\right] .
\end{gathered}
$$

It follows from (14), (16), and (7) that

$$
\begin{aligned}
& {\left[\begin{array}{cc}
{\widetilde{\Phi^{*}}}^{\prime}(x, \lambda) & -\widetilde{\Phi}^{*}(x, \lambda) \\
-{\widetilde{\varphi^{*}}}^{\prime}(x, \lambda) & \widetilde{\varphi}^{*}(x, \lambda)
\end{array}\right] \cdot\left[\begin{array}{cc}
\widetilde{\varphi}(x, \lambda) & \widetilde{\Phi}(x, \lambda) \\
\widetilde{\varphi}^{\prime}(x, \lambda) & \widetilde{\Phi}^{\prime}(x, \lambda)
\end{array}\right]} \\
& =\left[\begin{array}{ll}
I & 0 \\
0 & I
\end{array}\right] .
\end{aligned}
$$

Consequently,

$$
\begin{aligned}
& P_{11}(x, \lambda)=\varphi(x, \lambda) \widetilde{\Phi^{*}}(x, \lambda)-\Phi(x, \lambda) \widetilde{\varphi^{*}}(x, \lambda), \\
& P_{12}(x, \lambda)=\Phi(x, \lambda) \widetilde{\varphi}^{*}(x, \lambda)-\varphi(x, \lambda) \widetilde{\Phi}^{*}(x, \lambda) .
\end{aligned}
$$

Using (14) and (11) we obtain

$$
\begin{aligned}
& \varphi^{(\nu)}(x, \lambda) \\
& =-\frac{i}{2}(-i \rho)^{\nu} \exp (-i \rho x)[I]_{\beta} B_{12}^{0} \operatorname{diag}\left\{\rho^{-\mu_{1 q}}\right\}, \\
& \quad \rho \in \Omega_{\delta}, \quad \nu=0,1, x \in(0, T) .
\end{aligned}
$$

In order to study the asymptotic behavior of $\Phi(x, \lambda)$, introduce the matrix solution $\psi(x, \lambda)$ of (1) under the initial conditions at the regular end: $\psi(T, \lambda)=-I, \psi^{\prime}(T)=H$. Then $\Phi(x, \lambda)=\psi(x, \lambda)(U(\psi))^{-1}$. Using the standard asymptotic representation for $\psi(x, \lambda)$, one can expand this solution by the system $Y_{k}(x, \lambda), k=1,2$, and then calculate asymptotic for $U(\psi)$, applying (9) and (10). Finally, one gets the following result:

$$
\begin{aligned}
& \Phi^{(\nu)}(x, \lambda)=\exp (i \rho x)[I]_{\beta}\left(B_{12}^{0}\right)^{-1} \operatorname{diag}\left\{\rho^{-\mu_{2 q}}\right\}, \\
& \rho \in \Omega_{\delta}, \quad \nu=0,1, x \in(0, T) .
\end{aligned}
$$

Similarly one can obtain the asymptotic formulas for $\widetilde{\varphi}^{*}(x, \lambda)$ and $\widetilde{\Phi}^{*}(x, \lambda)$. Substituting all these asymptotic relations into (21), we get

$$
\begin{aligned}
& P_{11}(x, \lambda)=[I]_{\beta}, \\
& P_{12}(x, \lambda)=O\left(\rho^{-\beta}\right), \\
& x \in(0, T), \rho \in \Omega_{\delta} .
\end{aligned}
$$

It follows from (21), (14), and (16) that

$$
\begin{aligned}
P_{11}(x, \lambda)= & \varphi(x, \lambda) \widetilde{S_{2}^{*}}-S_{2}(x, \lambda) \widetilde{\varphi^{*}}(x, \lambda) \\
& +\varphi(x, \lambda)\left(\widetilde{M}^{*}(\lambda)-M(\lambda)\right){\widetilde{\varphi^{*}}}^{\prime}(x, \lambda), \\
P_{12}(x, \lambda)= & S_{2}(x, \lambda) \widetilde{\varphi}^{*}(x, \lambda)-\varphi(x, \lambda) \widetilde{S}_{2}^{*} \\
& +\varphi(x, \lambda)\left(M(\lambda)-\widetilde{M}^{*}(\lambda)\right) \widetilde{\varphi}^{*}(x, \lambda) .
\end{aligned}
$$


Since $M(\lambda)=\widetilde{M}(\lambda)=\widetilde{M}^{*}(\lambda)$, the matrix functions $P_{11}(x, \lambda)$ and $P_{12}(x, \lambda)$ are entire with respect to $\lambda$. Taking asymptotic formulas (24) into account, we conclude that $P_{11}(x, \lambda) \equiv I$, $P_{12}(x, \lambda) \equiv 0$. By virtue of $(19), \varphi(x, \lambda) \equiv \widetilde{\varphi}(x, \lambda), \Phi(x, \lambda)=$ $\widetilde{\Phi}(x, \lambda)$, and therefore $L=\widetilde{L}$.

\section{Conflict of Interests}

The author declares that there is no conflict of interests regarding the publication of this paper.

\section{Acknowledgments}

This work was supported by Grant 1.1436.2014K of the Russian Ministry of Education and Science and by Grants 13-0100134, 14-01-31042, and 15-01-04864 of Russian Foundation for Basic Research.

\section{References}

[1] Z. S. Agranovich and V. A. Marchenko, The Inverse Problem of Scattering Theory, KSU, Kharkov, Ukraine, 1960, English translation, Gordon and Breach, New York, NY, USA, 1963 (Russian).

[2] M. G. Gasymov, "Determination of St-L equation with a singular point from two spectra," Doklady Akademii Nauk SSSR, vol. 161, pp. 274-276, 1965, English translation: Soviet Mathematics-Doklady, vol. 6, pp. 396-399, 1965.

[3] V. A. Yurko, "An inverse problem for differential equations with a singularity," Differentsial'nye Uravneniya, vol. 28, no. 8, pp. 1355-1362, 1992 (Russian), English translation in Differential Equations, vol. 28, no. 8, pp. 1100-1107, 1992.

[4] G. Freiling and V. Yurko, "Inverse problems for differential operators with singular boundary conditions," Mathematische Nachrichten, vol. 278, no. 12-13, pp. 1561-1578, 2005.

[5] G. Freiling and V. Yurko, Inverse Sturm-Liouville Problems and Their Applications, Nova Science, Huntington, NY, USA, 2001.

[6] G. Freiling and V. Yurko, "Boundary value problems with regular singularities and singular boundary conditions," International Journal of Mathematics and Mathematical Sciences, vol. 2005, no. 9, pp. 1481-1495, 2005.

[7] V. A. Marchenko, Sturm-Liouville Operators and Their Applications, Naukova Dumka, Kiev, Ukraine, 1977, English translation: Birkhauser, 1986 (Russian). 


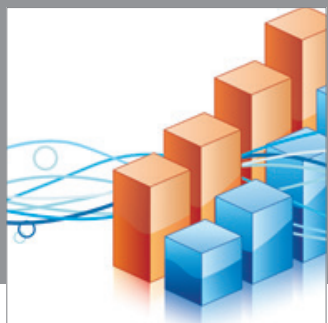

Advances in

Operations Research

mansans

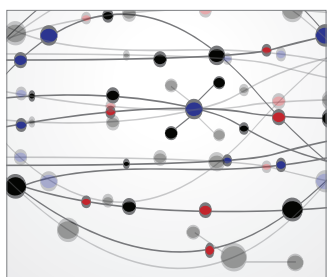

The Scientific World Journal
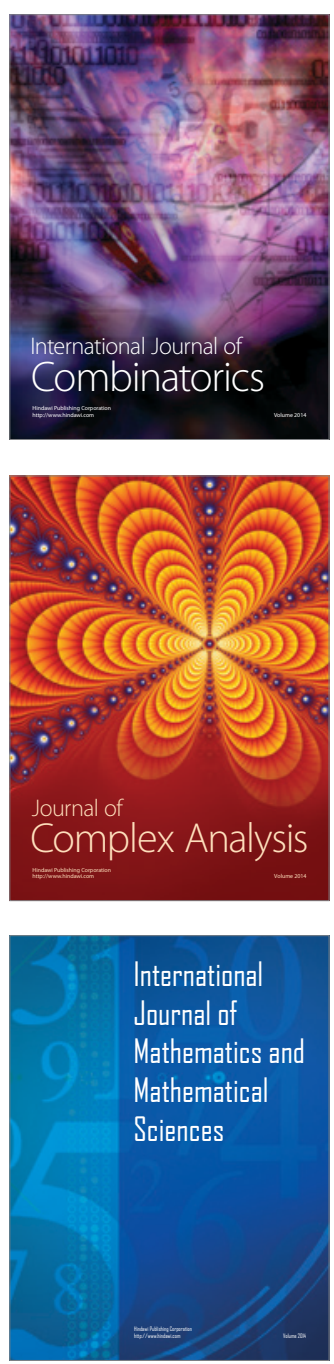
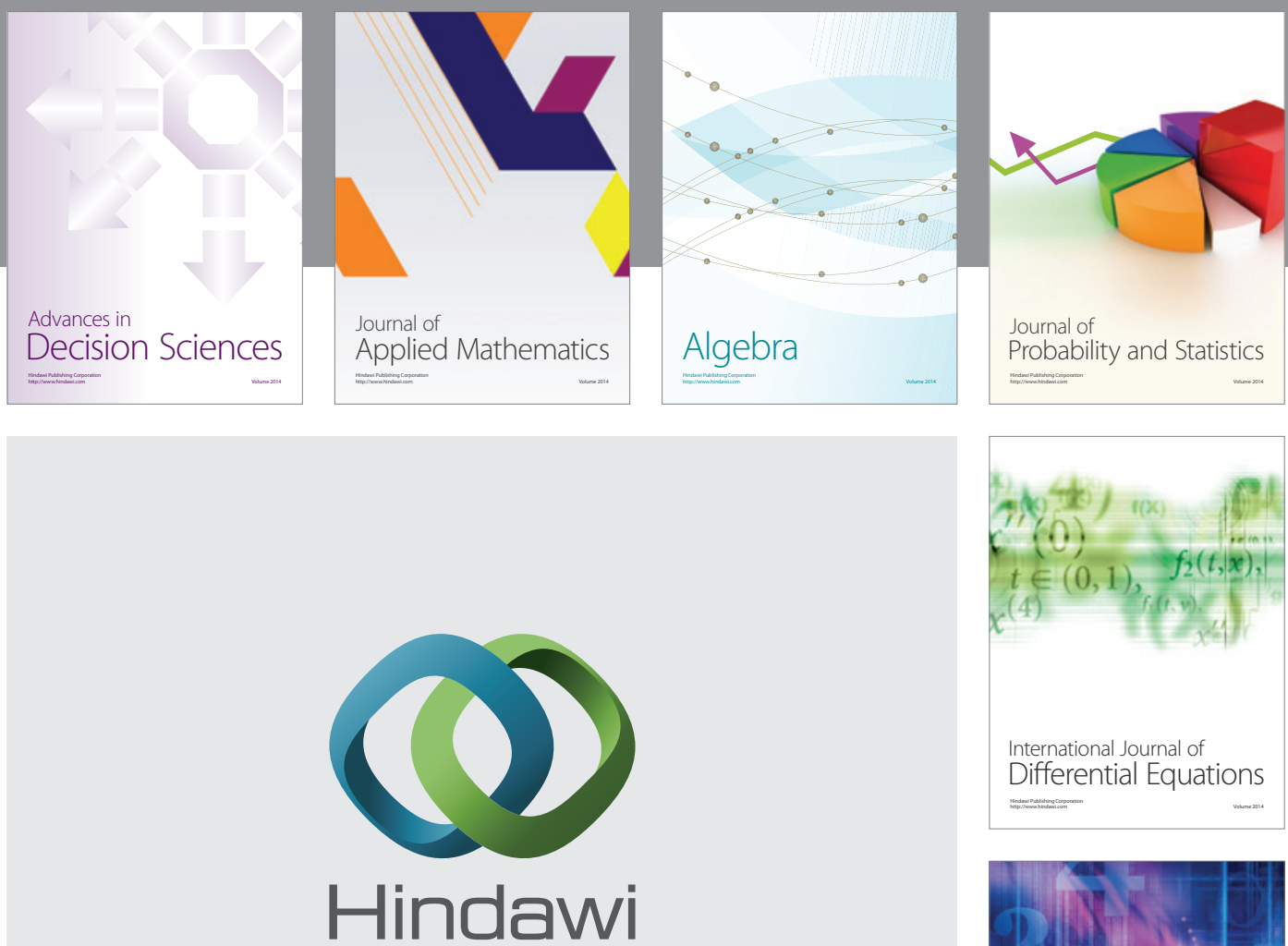

Submit your manuscripts at http://www.hindawi.com
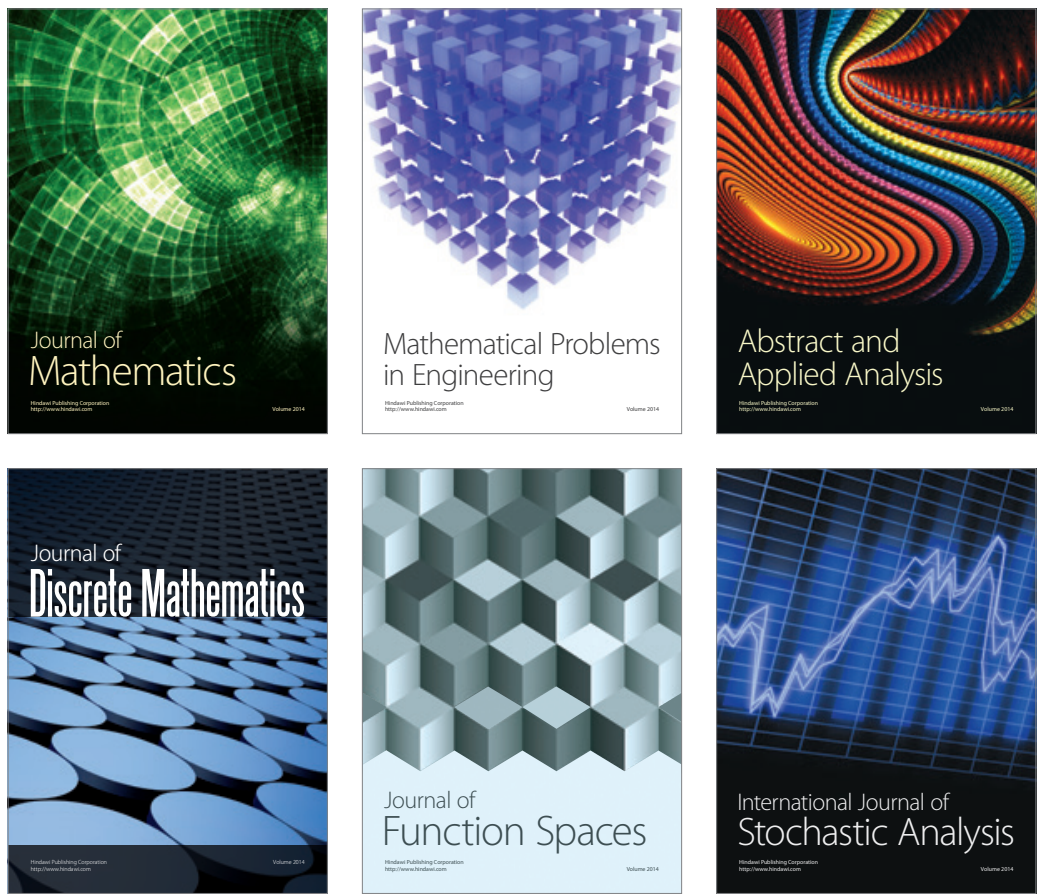

Journal of

Function Spaces

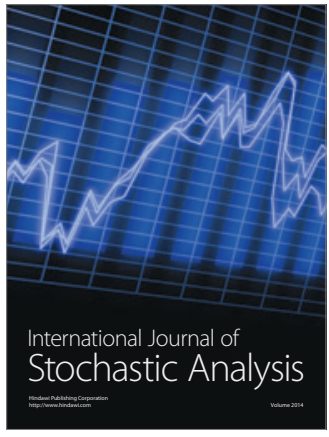

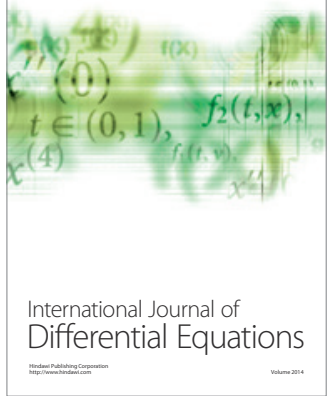
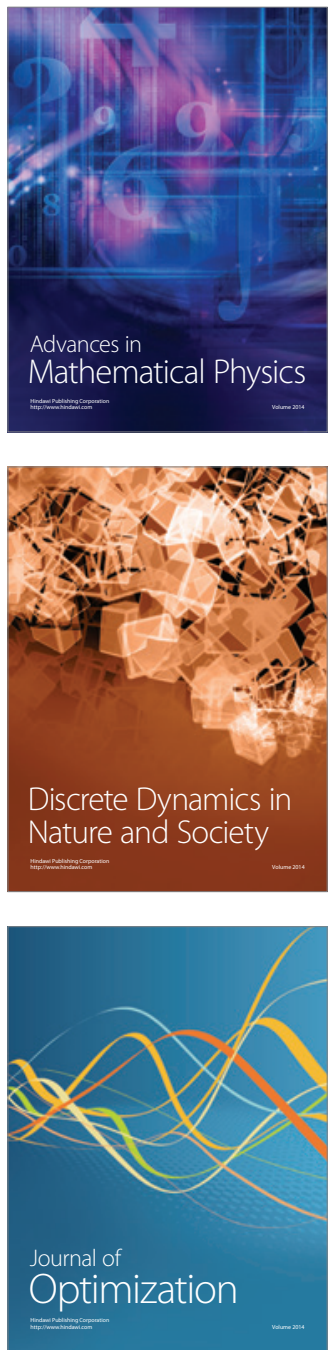